der Beweisführung vollständig folgen kann, und daß er einsehen kann, wie sich die Untersuchung auf alle durch Potenzreihen darstellbaren Funktionen ausdehnen läBt.

Die Anordnung der Tabellen habe ich so getroffen, $\mathrm{da} B$ die am häufigsten gebrauchten voranstehen, nämlich die fünfstelligen logarithmischen und trigonometrischen.

\title{
Vorwort zur dreiundzwanzigsten Auflage.
}

Seit der vierzehnten Auflage sind auf S. 36 einige Werte nach Angabe des Herrn Professor Mehler in Elbing und nach den Vorschlägen des Herrn Geheimrat Professor Förster in Berlin und des Herrn Professor Becker in Gotha die astronomischen Tabellen verbessert. In der neunzehnten ist im AnschluB an die gesetzliche Einführung der mitteleuropäischen Zeit eine Änderung und Erweiterung der Ortstafel vorgenommen worden.

Die jetzt vorliegende Auflage enthält eine Verbesserung der Tabelle $\nabla$ nach den Vorschlägen des Herrn P. Winnertz in Krefeld. Die Zahl der in die Abteilung A aufgenommenen Nantiffen konnte nämlich beträchtlich verkleinert werden, ohne den Zweck dieser Abteilung zu beeinträchtigen. Hierdurch ist es möglich geworden, die Mantiffen in A in ebenso großer Schrift zu setzen, wie die in B, was für das Ange eine große Erleichterung ist. Gleichzeitig sind, um die erreichbare Genauigkeit zu vermehren, jene Iantiffen achtstellig gegeben. Im Zusammenhange mit diesen Verbesserungen wurde in den Erläuterungen eine Ånderung der $\S \S 10-13$ erforderlich.

Berlin, im Januar 1900.

Der Herausgeber. 